\title{
Ecología trófica de la Sabaleta Brycon henni (Pisces: Characidae) en el río Portugal de Piedras, Alto Cauca, Colombia
}

\section{Trophic ecology of Brycon henni (Pisces: Characidae) in the Portugal de Piedras river, upper Cauca basin, Colombia}

\author{
Alvaro Botero-Botero, ${ }^{1,2 *}$ M.Sc, Hernán Ramírez-Castro, ${ }^{2}$ Lic.
}

\begin{abstract}
${ }^{1}$ Universidad Nacional Experimental de los Llanos "Ezequiel Zamora" - UNELLEZ (Guanare, Venezuela), Fundación Neotrópica-Colombia, La Tebaida, Quindío, Colombia. ${ }^{2}$ Universidad del Quindío, Programa de Licenciatura en biología y Educación ambiental. Pereira, Colombia. *Correspondencia: albotero33@ yahoo.com
\end{abstract}

Recibido: Mayo de 2010; Aceptado: Diciembre de 2010.

\section{RESUMEN}

Objetivo. Estudiar la ecología trófica de la sabaleta (Brycon henni) en el río Portugal de Piedras, cordillera Oriental, departamento del Valle del Cauca. Materiales y métodos. Desde octubre de 2008 hasta junio de 2009 se realizaron pescas exploratorias y se determinaron parámetros físico y químicos del hábitat. Los ejemplares capturados fueron eviscerados y el contenido estomacal fue determinado hasta el mínimo taxón posible. Resultados. La especie presenta una dieta generalista que incluye 35 categorías alimenticias, con tendencia al consumo de larvas y ninfas de insectos acuáticos entre los cuales se destacan tricópteros, dípteros y odonatos; también, consume organismos alóctonos al cauce como hormigas (Hymenoptera), escarabajos (Coleoptera) y material vegetal: frutos, semillas y hojas. La relación longitud intestino (LI) vs. longitud estándar (LS) indican que la especie presenta características propias de una especie carnívora (LI $=-13.8728+1.02377 *$ LS, $r=0.35, n=22$ ), a su vez, el peso total (PT) depende directamente de la longitud total (LT) y LS del pez (PT = -49.308 + 0.609962*LT; $r=0.92 \mathrm{n}=30 ;$ PT = -41.6011 + 0.672529*LS; $r=0.89, n=30$, respectivamente). Conclusiones. La sabaleta (Brycon henni) presentó caracteristicas de una especie carnivora.

Palabras clave: Brycon henni, ecología, dieta, Colombia. (Fuente: AIMS) 


\section{ABSTRACT}

Objetive. Study the trophic ecology of the sabaleta (Brycon henni) on the river Portugal de Piedras, from eastern range, department of Valle del Cauca. Materials and methods. During a 9 months period (October 2008 - June 2009), exploratory fishing, were carried out to determine the physical and chemical parameters of the habitat. The captured fishes were gutted, and the contents of the gut were determined to the nearest taxon. Results. The diet of the species included 35 alimentary categories, the trend leaned towards larvae and nymphs of aquatic insects of whom the most common species found were members of the Trichoptera, Diptera and Odonata; organisms allochthonous to the stream of the river sere also found to be part of the diet of the $B$. henni, such as ants (Hymenopterae), beetles (Choleopterae) and vegetal material such as fruits, leaves and seeds. The ratio between gut length Vs. Standard length indicates that the species presents the features of a carnivore species ( $\mathrm{LI}=-13.8728+1.02377 * \mathrm{LS}, \mathrm{r}=0.35, \mathrm{n}=22$ ), in turn the total weight depended directly on the total length and standard length of the fish (PT $=-49.308+0.609962 * \mathrm{LT} ; \mathrm{r}=$ $0.92 \mathrm{n}=30 ;$ PT $=-41.6011+0.672529 * \mathrm{LS} ; \mathrm{r}=0.89, \mathrm{n}=30$, respectively). Conclusions. The sabaleta Brycon henni, exhibited characteristics of a carnivorous species.

Key words: Brycon henni, ecology, diet, Colombia. (Sourse: AIMS)

\section{INTRODUCCIÓN}

El género Brycon, está constituido aproximadamente por cuarenta especies válidas $(1,2)$, once de las cuales han sido reportadas en los ríos Amazonas, Atrato, Cauca, Dagua, San Juan, Sinú, Magdalena, Meta, Mira, Orinoco y Patía en Colombia (1). Es considerado como un género monofilético (3), con especies relativamente grandes dentro de los carácidos, de amplia distribución en ríos de Centro América, y en cuencas Suramericanas, como Paraná, Orinoco y Amazonas $(1,4)$.

Brycon henni, es una de las especies más importantes de los pequeños ríos que nacen en la cordillera Central de Colombia (2). La especie se distribuye en la región central de Colombia en el Alto y Bajo río Cauca, así como en el río Magdalena, en la región pacifica en los ríos San Jorge y San Juan $(5,6)$; en el río Cauca su distribución se restringe al rango 1000-1500 msnm (7). En la zona andina es factible discriminar dos grupos, por características genéticas, los de la cuenca del río Cauca y los de la cuenca del río Magdalena (2). B. henni es una de las especies más abundantes dentro de los ríos de alta montaña en la cuenca del río Cauca $(8,9,10)$ y es una de las preferidas para la pesca deportiva y de subsistencia en la zona.
El objetivo del estudio fue determinar la ecología trófica de $B$. henni en el río Portugal de Piedras, cordillera oriental del departamento del Valle del Cauca.

\section{MATERIALES Y MÉTODOS}

Sitio de estudio. Para la captura de los peces, se realizaron siete salidas de campo, desde octubre de 2008 hasta junio de 2009, a la zona media y baja del río Portugal de Piedras, afluente del río Cauca, (403' 635", 76 21' 117"; 1012msnm \& $4^{\circ} 03^{\prime} 722^{\prime \prime} \mathrm{N}, 7^{\circ} 18^{\prime} 915^{\prime \prime}$ O; $963 \mathrm{msnm}$ ).

Muestreos y tipo de captura de especimenes. Los muestreos abarcaron los períodos de altas lluvias (octubre, noviembre y diciembre) y de bajas lluvias (enero, febrero, marzo y junio). Los peces fueron colectados con atarraya, con un esfuerzo de muestreo de dos hombres durante una (1) hora, es de resaltar que, en los meses de bajas Iluvias la captura de individuos fue baja.

Conservación y procesamiento de especimenes. Los peces fueron preservados en hielo para su traslado al laboratorio de 
Biología de la Universidad del Quindío. Se capturaron y examinaron 39 ejemplares los cuales fueron medidos, pesados y se les practicó una disección uroventral para la extracción del tracto digestivo. Se encontraron 39 estómagos llenos, los cuales fueron medidos y sus ciegos pilóricos contados.

Analisís de contenido estomacal. Los contenido estomacales fueron analizados por medio de los métodos de ocurrencia, numérico $(11 ; 12)$, volumétrico $(13,14)$ e índice de importancia (15).

Caracterización del hábitat. Para la caracterización del hábitat de la especie se colectaron muestras de agua en frascos plásticos de un litro, durante los periodos lluvias altas (desde Septiembre hasta noviembre de 2008) y en lluvias bajas (desde diciembre del 2008 hasta febrero del 2009). En el laboratorio se determinaron las siguientes variables: demanda bioquímica de oxigeno (DBO), titulando oxigeno disuelto pasado cinco días por el método Winkler, alcalinidad y sólidos disueltos, oxígeno disuelto y $\mathrm{pH}$ se determinaron por titulación de acuerdo a métodos estándares (16). Además, in situ fueron medidas variables físicas del cauce tales como ancho, profundidad, tipo de sustrato y temperatura del aire y del agua.

Analisís estadísticos. Fueron realizadas correlaciones lineales entre la longitud del intestino vs. Longitud estándar, el peso del animal vs. la longitud estándar y la longitud total.

\section{RESULTADOS}

Características del hábitat. El río Portugal de Piedras es un río de tipo terciario ubicado en el municipio de Ríofrío, departamento del Valle del Cauca en el costado oriental de la cordillera occidental. Presenta riberas bien conservadas en la parte alta, pero en la zona media y baja están dominadas por cultivos de caña de azúcar y pastos para la ganadería.

En la zona media y baja el río presentó un ancho promedio de $11.1 \mathrm{~m}$ en lluvias altas y disminuye a $7.7 \mathrm{~m}$ en bajas lluvias, y una profundidad media de 0.84 en lluvias altas y 0.69 en bajas Iluvias. El sustrato compuesto de textura mixta, conformado principalmente por arena, cantos rodados, y materia orgánica en descomposición. El agua es cristalina o clara, no en tanto, al presentarse lluvias se torna café oscuro. La temperatura del aire fue de $22.5^{\circ} \mathrm{C}$ en lluvias altas y en lluvias bajas de $25^{\circ} \mathrm{C}$, y la temperatura del ambiente fue de $20.6^{\circ} \mathrm{C}$ en lluvias altas y $23.6^{\circ} \mathrm{C}$ en Iluvias bajas. El oxígeno disuelto fue medio de $6.1 \mathrm{mg} / \mathrm{l}$ en lluvias altas y disminuyó cuando en la estación de bajas lluvias

Tabla 1. Variables físicas y químicas de dos temporadas climáticas, en el río Portugal de Piedras, Alto Cauca, Colombia.

\begin{tabular}{lcc}
\hline \multicolumn{1}{c}{ variable/temporada } & $\begin{array}{c}\text { Lluvias altas } \\
\text { (Sep-nov 2008) } \\
\text { (Promedio) }\end{array}$ & $\begin{array}{c}\text { Lluvias bajas } \\
\text { (Dic. 08-feb 09) } \\
\text { (Promedio) }\end{array}$ \\
\hline Profundidad (m) & 0.84 & 0.69 \\
Ancho del cauce $(\mathrm{m})$ & 11.1 & 7.7 \\
Temperatura del agua $\left({ }^{\circ} \mathrm{C}\right)$ & 20.6 & 23.6 \\
Temperatura del aire $\left({ }^{\circ} \mathrm{C}\right)$ & 22.5 & 25 \\
Velocidad de la corriente $(\mathrm{m} / \mathrm{s})$ & 0.56 & 0.37 \\
Sólidos disueltos (mg/l) & 162 & 140 \\
Sólidos suspendidos $(\mathrm{mg} / \mathrm{l})$ & 7.5 & 5.2 \\
Alcalinidad (mg/l CaCO3) & 59 & 71 \\
Oxígeno disuelto $(\mathrm{ml} / \mathrm{l})$ & 6.1 & 5.8 \\
DBO (mg/l) & 1.45 & 8.3 \\
DQO (mg/l) & 457 & 465 \\
pH & 8.3 & 7.2 \\
\hline
\end{tabular}


5.8. El pH presentó valores cercanos a la neutralidad (7.2) en la estación de lluvias bajas, mientras que con el incremento de las lluvias este también incremento 8.3. La DQO permaneció estable en ambos periodos climáticos, mientras que la DBO incrementó en el periodo de bajas lluvias (Tabla 1).

Aspectos morfométricos. Los individuos capturados fueron en general peces grandes con una longitud estándar media de $115.8 \mathrm{~mm}$ (valor mínimo: 88 y valor máximo: $152 \mathrm{~mm}$ ).

Brycon henni posee un estómago más largo (media $25.7 \mathrm{~mm}$; Ds 9.5) que ancho (media $13.4 \mathrm{~mm}$; Ds 12.85), el cual se ubica en la parte anterior de la cavidad celómica en medio de la masa visceral. Los ciegos pilóricos se localizan en la parte anterior del estómago siendo casi tan largos como el estomago y su número promedio fue de 13 (dato mínimo 11, dato máximo 16).

Positiva y baja correlación fue hallada entre la longitud del intestino vs. longitud estándar (Longitud del intestino $=-13.8728+1.02377 *$ LS, $\mathrm{r}=0.35, \mathrm{n}=22)$, mientras el peso total vs longitud total presentó alta correlación (Peso $=-49.308+$ $0.609962 * \mathrm{LT} ; \mathrm{r}=0.92 \mathrm{n}=30$ ), al igual que el peso

Tabla 2. Contenido estomacal de Brycon henni en el río Portugal de Piedras, Alto Cauca, Colombia. A: Adultos, L: Larvas, N: Ninfas.

\begin{tabular}{|c|c|c|c|c|}
\hline Contenido & $\begin{array}{c}\% \\
\text { numerico } \\
\end{array}$ & $\begin{array}{c}\% \\
\text { volumen } \\
\end{array}$ & $\begin{array}{c}\% \\
\text { frecuencia } \\
\end{array}$ & $\begin{array}{c}\text { Índice de } \\
\text { importancia }\end{array}$ \\
\hline Hymenoptera. Formicidae (A) & 2.88 & 0.89 & 20.00 & 0.178 \\
\hline Hymenoptera. vespidae (A) & 1.08 & 2.05 & 8.57 & 0.176 \\
\hline Ephermeroptera (L) & 0.36 & 0.02 & 2.86 & 0.001 \\
\hline Ephermeroptera. Baetidae (N) & 0.36 & 0.07 & 2.86 & 0.002 \\
\hline Odonata $(\mathrm{N})$ & 0.36 & 0.15 & 2.86 & 0.004 \\
\hline Odonata. Libellulidae (N) & 0.36 & 1.39 & 2.86 & 0.040 \\
\hline Coleoptera. Elmidae (L) & 0.72 & 0.15 & 5.71 & 0.008 \\
\hline Coleoptera. Psephenidae (L) & 0.36 & 0.15 & 2.86 & 0.004 \\
\hline Coleoptera (A) & 1.80 & 19.33 & 14.29 & 2.761 \\
\hline Trichoptera. Helicosychidae (L) & 3.96 & 0.39 & 5.71 & 0.022 \\
\hline Trichoptera. Hydroptilidae (L) & 1.08 & 0.18 & 5.71 & 0.011 \\
\hline Trichoptera. Letoceridae (L) & 5.04 & 1.74 & 11.43 & 0.199 \\
\hline Diptera. Ceratopogonidae (L) & 0.72 & 0.11 & 5.71 & 0.006 \\
\hline Diptera. Culicidae (L) & 0.36 & 0.07 & 2.86 & 0.002 \\
\hline Diptera. Cyclorrapha (L) & 0.36 & 0.04 & 2.86 & 0.001 \\
\hline Diptera. Empididae (L) & 0.36 & 0.15 & 2.86 & 0.004 \\
\hline Diptera. Dixidae (L) & 0.36 & 0.15 & 2.86 & 0.004 \\
\hline Diptera. Chironomidae (L) & 0.36 & 0.04 & 2.86 & 0.001 \\
\hline Diptera. Tipulidae (L) & 0.36 & 0.22 & 2.86 & 0.006 \\
\hline Diptera (A) & 0.72 & 0.13 & 5.71 & 0.007 \\
\hline Diptera.Brachycera (A) & 0.36 & 0.09 & 2.86 & 0.003 \\
\hline Restos de Insectos & 67.27 & 25.36 & 40.00 & 10.144 \\
\hline nematodos & 0.36 & 0.07 & 2.86 & 0.002 \\
\hline Aranea & 0.36 & 0.17 & 2.86 & 0.005 \\
\hline Algas. Chlorophyta & 0.00 & 21.00 & 14.29 & 2.999 \\
\hline Materia vegetal. Hojas & 0.00 & 2.77 & 8.57 & 0.238 \\
\hline Material vegetal. Semillas & 3.60 & 1.15 & 14.29 & 0.164 \\
\hline Material vegetal. frutos & 5.40 & 14.13 & 11.43 & 1.615 \\
\hline Material vegetal & 0.00 & 7.55 & 2.86 & 0.216 \\
\hline Escama de Characiformes & 0.36 & 0.15 & 2.86 & 0.004 \\
\hline piedras & 0.36 & 0.15 & 2.86 & 0.004 \\
\hline Material digerido & 0.00 & 34.61 & 40.00 & 13.844 \\
\hline
\end{tabular}


total vs. longitud estándar (Peso $=-41.6011+$ $0.672529 *$ LS; $r=0.89, \mathrm{n}=30$ ).

Características de la dieta. El contenido estomacal se agrupó en 35 ítems (Tabla 2). Los organismos consumidos en mayor cantidad (análisis numérico) fueron los insectos acuáticos en especial los Trichoptera, Letoceridae y Helicosychidae, y seguido por partes de insectos y material de origen alóctono como Hymenoptera, Formicidae, y frutos; los ítem que más volumen ofrecieron a la dieta fueron: Algas, Chlorophyta, Coleopteras adultos de origen alóctono, frutos y restos de insectos (Tabla 3 ), dentro de los ítem que mas frecuencia de aparición en la dieta de la sabaleta se encontraron: Hormigas, escarabajos de origen externo a la columna de agua, algas, semillas, frutos, Trichoptera, Letoceridae y restos de insectos. Los ítems más importantes fueron: restos de insectos, Coleópteros adultos, Algas y frutos (Tabla 2).

\section{DISCUSIÓN}

El espectro alimenticio sobre el que los animales seleccionan su dieta es enorme, por lo que suelen restringir sus pesquisas a una gama mucho menor de posibilidades en función de las ofertas coyunturales del hábitat que ocupen y de sus propias adaptaciones (17).

Los peces han adoptado una gran cantidad de rutas para obtener su alimento y conocer la energía y sus requerimientos nutricionales. Como los vertebrados acuáticos ellos encuentran un potencial de ítem alimenticio, algunos de estos ítems flotan microscópicamente desde diferentes profundidades, otros enterrados sobre el lecho del río, adheridos al sustrato, otros nadan libremente y algunos ocultos en grietas y cuevas y otros que caen eventualmente al agua desde las zonas ribereñas.

Brycon henni es una especie omnívora oportunista que consume recursos tanto del fondo del agua como larvas de dípteros y tricópteros y elementos de origen externo al cauce como hormigas, escarabajos, avispas. Además de esta dieta carnívora, la especie incorpora a su alimentación ítems de origen vegetal como semillas, hojas y frutos. Iguales resultados han sido reportados para $B$. henni $(18,19)$, B. falcatus, $B$. melanopterus y $B$. opalinus (20) pareciendo ser esta tendencia trófica una generalidad para el género.

Aunque los resultados de la relación longitud intestino vs. Iongitud estándar muestran una tendencia diferente, indicando que la sabaleta presenta características propias de una especie carnívora puesto que las especies herbívoras tienden a registrar un intestino más largo que las carnívoras (21). La relación en carnívoros es menor que uno (22).

La presencia de abundantes ítem de origen alóctono entre los cuales se encuentran frutos, semillas, e insectos demuestran la importancia de los bosque ribereños para los arroyos pequeños de alta montaña, pues en este tipo de arroyos las aguas son claras y transparentes, pobres en nutrientes y de baja conductividad, presentan temperaturas bajas y la producción primaria es muy baja o nula (23), dependiendo los consumidores de material alóctono que cae de los árboles o que es arrastrado por la lluvias. $(24,23)$.

Aunque algunas especies del genero Brycon pueden considerarse como dispersoras de semillas (25) para el caso de $B$. henni se debe tomar con precaución este supuesto, puesto que la mayorías de semillas encontradas dentro del estomago presentaban algún daño mecánico (degradación, trituración) que las pueden inhabilitar en su germinación, para sacar conclusiones solidas.

Respecto a la preferencia de hábitat los resultados del presente estudio coinciden con los planteados por Builes y Uran (26) pues la especie está presente en arroyos con concentraciones altas de oxigeno (7-10 mg./l), prefiriendo aguas 
claras y ubicándose en el día en las zonas finales de los charcos para la captura de alimentos.

En conclusión, se puede afirmar que la Sabaleta Bricon henni, presentó caracteristicas que la ubican como una especie carnivora.

\section{Agradecimientos}

\begin{abstract}
A John D. y Catherine T. MacArthur Foundation a través del programa Colombia de WCS, Fundación Neotrópica-Colombia y al Laboratorio de Biología de la Universidad del Quindío por aportar equipos y recursos para la realización de este proyecto. A Jhon Anderson Arcila, por su colaboración en trabajo de campo a Julián Andrés Sánchez-Pachón por su colaboración en el trabajo de laboratorio.
\end{abstract}

\section{REFERENCIAS}

1. Howes G. Review of the genus Brycon (Teleostei, Characoidei). Bull $\mathrm{Br}$ Mus (Nat Hist) Zool 1982; 43:1-47.

2. Pineda HS, Arboleda L, Echeverry A, Urcuqui ES, Molina D, Olivera M. et al. Caracterización de la diversidad genética en el pez Brycon henni (Characiformes: Characidae) en Colombia central por medio de marcadores RAPD. Rev Biol Trop 2007; 55(3-4):1025-1035.

3. Malabarba M. Phylogeny of fossil Characiformes and paleobiogeography of the Tremenbé formation. Sao Paulo, Brazil. In: Malabarba LR et al. (eds). Phylogeny and classification of neotropical fishes. Porto Alegre, Brasil: Edipucrs; 1998.

4. Royero LR. Peces de Venezuela. Valencia, Estado Carabobo. Venezuela: Edición Raúl Clemente. C.A; 1992.

5. Dahl G. Los peces del norte de Colombia. Bogotá: Inderena: 1971.

6. Maldonado-Ocampo JA, Ortega-Lara A, Usma-Oviedo JS. Peces de los Andes de Colombia. Bogotá : Instituto HUMBOLDT Colombia; 2005.
7. Ortega-Lara A, Usma J, Bonilla P, Santos N. Peces de la cuenca alta del río Cauca, Colombia. Biota Colombiana 2006; 7(1):39-54.

8. Jiménez J., Román-Valencia C., \&, Cardona, M. Distribución y constancia de las comunidades de peces del río San Pablo, Cuenca del río la Paila, Alto Cauca, Colombia. Revista Actualidades Biológicas 1998; 20:(68). 21-27.

9. Cardona M., C., Román-Valencia, J., Jiménez J., \& H., Hurtado. Composición y diversidad de la quebrada San Pablo en Alto Cauca, Colombia. Boletín Ecotrópica 1998; (32):11-24.

10. García-Álzate R., A., C., García Álzate \& Botero-Botero Composición, estacionalidad y hábitat de los peces de la Quebrada Cristales, afluente del río La Vieja, Alto Cauca, Colombia. Revista de Investigaciones Universidad del Quindío 2010; (19):115-121.

11. Hynes $H$. The food of fresh-water Sticklebacks (Gasterosteus aculeatus and Pygosteus pungitius) with a review of methods used in studies of the food of fishes. J Anim Ecol 1950; 19:36-58. 
12. Hyslop EJ. Stomach contents analysis- a review and methods and their application. J Fish Biol 1980; 17(3):411-429.

13. Pedley R. \& Jones J.. The comparative feeding behaviour of brown trout, Salmon trutta L. and Atlantic Salmon, Salmon salar L. in Llyn Dwythwch, Wales. J Fish Biol 1978; 12:253-256.

14. Capitoli RR. Métodos para estimar volúmenes do conteudo alimentar de peixes e macroinvertebrados-Atlantica, Rio Grande, 1992; 4:117-120.

15. Oda D, J Parrish. Ecology of commercial snappers and groupers introduced to Hawaiian reefs. Proc. fourth International Coral Reef Symp 1981; 1:59-67.

16. Apha Awwa Wpcf. Métodos normalizados para el análisis de aguas potables y residuales. 28006 Madrid España: Ediciones Días y Santos, Juan Bravo; 1992.

17. Telleria JL. Zoología evolutiva de los vertebrados. Madrid: Editorial síntesis; 1987.

18. Román-Valencia C. Composición y estructura de las comunidades de peces de la cuenca del río La Vieja, Alto Cauca, Colombia. Biología y Educación 1993; 3(5):8-19.

19. Usma J., S. Estudio preliminar de la ictiofauna del río Escalerete. Cespedesia 1996; 21(68):41-53.
20. Zuntini DV, Wagner F, Dos Santos, Pereira S, Guimarães E. Alimentação natural da Piraputanga, Brycon hilarii (Teleostei- Characidae) no Rio Miranda, Município de Jardim, MS - Projeto Miracema . Brasil: IV simposio sobre recursos naturais e Sócio-economicos do Pantanal Corumbá/MS; 2006.

21. Kramer D, Bryant, M. Intestine length in the fishes of a tropical streams: ontogenetic allometry. Environ Biol Fishes 1995; 42:115-127.

22. Bussing, A. Fish communities and environmental characteristics of a tropical rain forest river in Costa Rica. Rev Biol Trop 1993; 4:791-809.

23. Dajoz, R. Tratado de ecología. $2^{a}$ edición. Madrid: Ed. Mundi-Prensa; 2002.

24. Roldan PG. Fundamentos de Limnología neotropical. Medellín Colombia: Editorial Universidad de Antioquia; 1992.

25. Reys, P., Sabino J., \& Galetti M. Frugivory by the fish Brycon hilarii (Characidae) in western Brazil. Acta Oecologica 2008; 35(1):136-141.

26. Builes J., Uran A. Estudio del cíclo sexual de la Sabaleta Brycon henni. Su comportamiento y fecundación artificial. Revista Actualidades Biológicas 1974; 3:2-12. 